\title{
Phase-referenced interferometer with subwavelength and subhertz sensitivity applied to the study of cell membrane dynamics
}

\author{
Changhuei Yang, Adam Wax, Mariah S. Hahn, Kamran Badizadegan,* \\ Ramachandra R. Dasari, and Michael S. Feld \\ George Harrison Spectroscopy Laboratory, Massachusetts Institute of Technology, Cambridge, Massachusetts 02139
}

Received March 28, 2001

\begin{abstract}
We report a highly sensitive means of measuring cellular dynamics with a novel interferometer that can measure motional phase changes. The system is based on a modified Michelson interferometer with a composite laser beam of 1550-nm low-coherence light and 775-nm CW light. The sample is prepared on a coverslip that is highly reflective at $775 \mathrm{~nm}$. By referencing the heterodyne phase of the 1550-nm light reflected from the sample to that of the 775-nm light reflected from the coverslip, small motions in the sample are detected, and motional artifacts from vibrations in the interferometer are completely eliminated. We demonstrate that the system is sensitive to motions as small as $3.6 \mathrm{~nm}$ and velocities as small as $1 \mathrm{~nm} / \mathrm{s}$. Using the instrument, we study transient volume changes of a few (approximately three) cells in a monolayer immersed in weakly hypotonic and hypertonic solutions. (C) 2001 Optical Society of America

OCIS codes: $050.5080,110.4500,180.3170,170.1530$.
\end{abstract}

Detection of motions with amplitude less than an optical wavelength and (or) velocity less than $1 \mu \mathrm{m} / \mathrm{s}$ is important for the study of subcellular dynamics in living biological tissues. Motions of such magnitude include the actin-based transport of organelles within the cells,${ }^{1}$ the ruffling of cell membranes, ${ }^{2}$ and the reaction of cell volumes to hypertonicity or hypotonicity. $^{3}$ These motions are of significant biomedical interest, and their study can potentially lead to new diagnostic modalities. For example, it has been established that cells with high metastatic potential are significantly more motile than normal cells. ${ }^{4}$ Cell dynamics is also of particular interest in the study of cell volume regulation mechanisms, which are identified by measurement of cell volume changes. ${ }^{3}$

We report a novel low-coherence phase-referenced interferometer that is capable of measuring slow $(\sim 1-100 \mathrm{~nm} / \mathrm{s})$ and small (4 $\mathrm{nm}$ or more) motions. In addition, the interferometer provides depth resolution and can be used for tomographic imaging. We verify the capability of the system by measuring the motion of a controlled piezoelectric transducer (PZT) and demonstrate its biological relevance in a cell volume regulation experiment.

Motional phase-referenced interferometry (PRI) differs from the widely applied Doppler optical coherence tomography (OCT) technique ${ }^{5,6}$ in that it is insensitive to mechanical jitter of the interferometer, which sets the lower sensitivity limit on Doppler OCT. PRI employs a noise-cancellation method similar to that used in phase-dispersion optical tomography (PDOT) ${ }^{7}$ The technique presented here differs from PDOT in that one of the two-low coherence sources is replaced with a continuous-wave $(\mathrm{CW})$ source. This modification results in an improved sensitivity to length changes $\left(\sim 10^{-2}\right.$ of an optical wavelength).

The experimental setup is based on a modified PDOT system, shown in Fig. 1. The input light is a two-color composite beam composed of CW light from a Ti:sapphire laser at $775 \mathrm{~nm}$ and low-coherence light (bandwidth, $40 \mathrm{~nm}$ ) from a 1550-nm Optospeed superluminescent diode. The composite beam is divided in two at the beam splitter. One part (signal) is brought to a focus at the target sample, while the other is incident upon a reference mirror moving at $0.5 \mathrm{~mm} / \mathrm{s}$, which induces a Doppler shift on the reference beam. The technique requires a strong reflection at the $\mathrm{CW}$ wavelength from a control interface at the sample. We fulfill this requirement by placing the sample on a glass coverslip; the index mismatch between the first glass interface and air is sufficient to provide the strong reflection. The powers of the 775- and 1550-nm beam components at the sample are 0.9 and $1.2 \mu \mathrm{W}$, respectively. An IR objective lens (12-mm focal length) focuses the composite beam onto the sample with a FWHM of $\sim 17 \mu \mathrm{m}$ at $1550 \mathrm{~nm}$. The focusing effect of the lens on the CW beam is not important, as we do not rely on this wavelength for imaging.

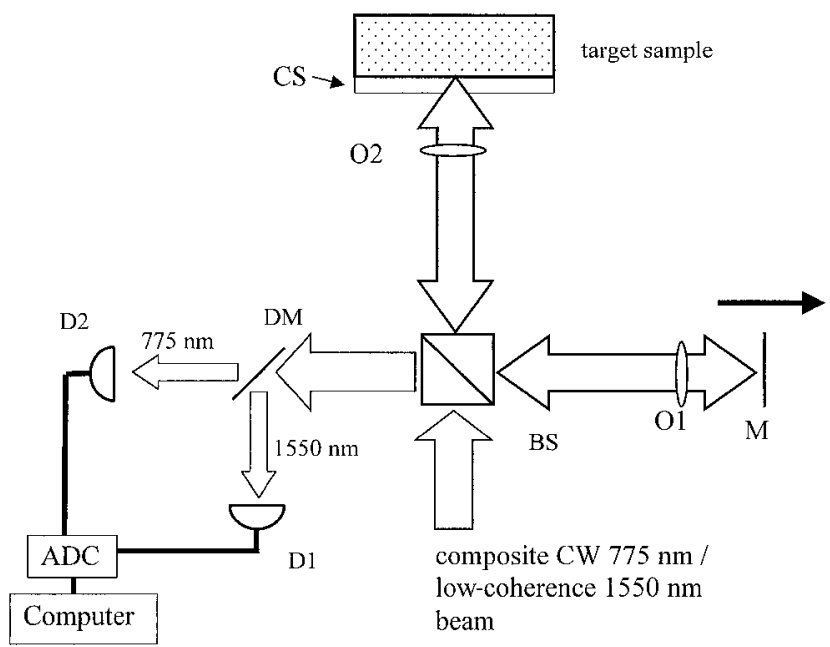

Fig. 1. Experimental setup: M, reference mirror; BS, beam splitter; O1, O2, microscope objectives; D1, D2, photodetectors; DM, $775 \mathrm{~nm} / 1550 \mathrm{~nm}$ dichroic mirror; CS, coverslip; ADC, analog-digital converter. 
The backreflected beams are recombined at the beam splitter, separated by their wavelength components by use of a dichroic mirror, and measured separately by photodetectors. The resulting heterodyne signals at their respective Doppler-shifted frequencies are recorded by means of a $16-$ bit $100-\mathrm{kHz}$ analog-digital converter. The signals are bandpassed around their respective center heterodyne frequencies and Hilbert transformed to extract their corresponding phases of the heterodyne signals, $\Psi_{\mathrm{CW}}$ and $\Psi_{\mathrm{LC}}$, are extracted. ${ }^{7}$ (The subscripts CW and LC are used throughout to denote the 775-nm CW and the 1550-nm low-coherence wavelength components, respectively).

The principle of PRI can be illustrated by a case in which the sample consists of two interfaces, 1 and 2 . The separation between them is $L$, and the refractive index of the material between them is $n$. The rate of change of the path-length difference between the reference and the signal arms is denoted $v$, which includes both the translation velocity of the reference mirror and the jitter of the interferometer. The phase of the $\mathrm{CW}$ heterodyne signal is

$$
\begin{aligned}
\psi_{\mathrm{CW}}(t)= & \bmod _{2 \pi}\left(\operatorname { a r g } \left\{R_{\mathrm{CW}, 1} \exp \left(i k_{\mathrm{CW}} 2 \int_{0}^{t} v \mathrm{~d} t\right)\right.\right. \\
& \left.\left.+R_{\mathrm{CW}, 2} \exp \left[i k_{\mathrm{CW}} 2\left(\int_{0}^{t} v \mathrm{~d} t-n_{\mathrm{CW}} L\right)\right]\right\}\right) \\
\cong & \bmod _{2 \pi}\left\{\arg \left[R_{\mathrm{CW}, 1} \exp \left(i k_{\mathrm{CW}} 2 \int_{0}^{t} v \mathrm{~d} t\right)\right]\right\} \\
= & \bmod _{2 \pi}\left(k_{\mathrm{CW}} 2 \int_{0}^{t} v \mathrm{~d} t\right),
\end{aligned}
$$

where $R_{\mathrm{CW} j}$ is the reflectivity of the interface $j$ at the $\mathrm{CW}$ wavelength and $k$ is the optical wave number. The factors of 2 in the exponentials are due to the effective doubling of optical paths in the backreflection geometry. The approximation is valid, provided that the first interface is a strong reflector compared with the second interface $\left(R_{\mathrm{CW}, 1} \gg R_{\mathrm{CW}, 2}\right)$.

The situation is different for the low-coherence light. When $\int_{0}^{t} v \mathrm{~d} t \approx n_{\mathrm{LC}} L$ and $L$ is much larger than the coherence length, the LC heterodyne signal will be dominated by the contribution from the light reflected by the second interface, since the contribution from the light reflected by the first interface is suppressed by coherence gating. The resulting LC heterodyne phase is then given by

$$
\begin{aligned}
\psi_{\mathrm{LC}}(t)= & \bmod _{2 \pi}\left(\operatorname { a r g } \left\{R_{\mathrm{LC}, 2} \exp \left[i k_{\mathrm{LC}} 2\left(\int_{0}^{t} v \mathrm{~d} t-n_{\mathrm{LC}} L\right)\right]\right.\right. \\
& \left.\left.\times \exp \left[-a 2\left(\int_{0}^{t} v \mathrm{~d} t-n_{\mathrm{LC}} L\right)^{2}\right]\right\}\right) \\
= & \bmod _{2 \pi}\left[k_{\mathrm{LC}} 2\left(\int_{0}^{t} v \mathrm{~d} t-n_{\mathrm{LC}} L\right)\right]
\end{aligned}
$$

with $a=4 \ln (2) /($ coherence length). Given that the center wavelength of the low-coherence source is chosen to be twice that of the CW source $\left(2 k_{\mathrm{LC}}=k_{\mathrm{CW}}\right)$, we can obtain a difference phase, $\psi_{D}$, that is simply a function of $L$ :

$$
\psi_{D}=\psi_{\mathrm{CW}}-2 \psi_{\mathrm{LC}}=\bmod _{2 \pi}\left(4 k_{\mathrm{LC}} n_{\mathrm{LC}} L\right) .
$$

This subtraction yields the phase acquired by the low-coherence light wave as it traverses from interface 1 to interface 2 and back. The effect of interferometer jitter, manifested as variations in $v$, is completely eliminated. By periodically scanning the sample and measuring this phase difference, we can determine changes in $L$ accurately. As the uncertainty in phase is $\sim 4.5^{\circ}$, we can measure time-dependent changes in $L$ with an accuracy of $\sim 3.6 \mathrm{~nm}$.

To establish the sensitivity of motional PRI for measurement of small and slow length changes, we study the motion of a PZT (Thorlabs AE0203D04) firmly mounted above a glass coverslip, with a thin layer of water between them [Fig. 2(a)]. The PZT is driven

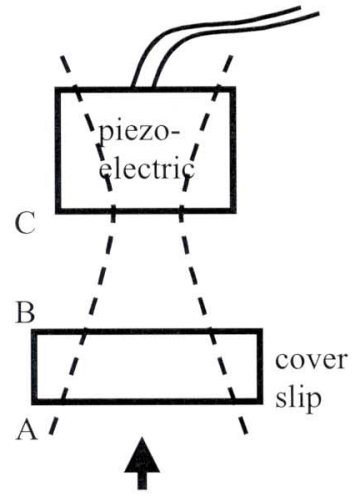

input light

(a)

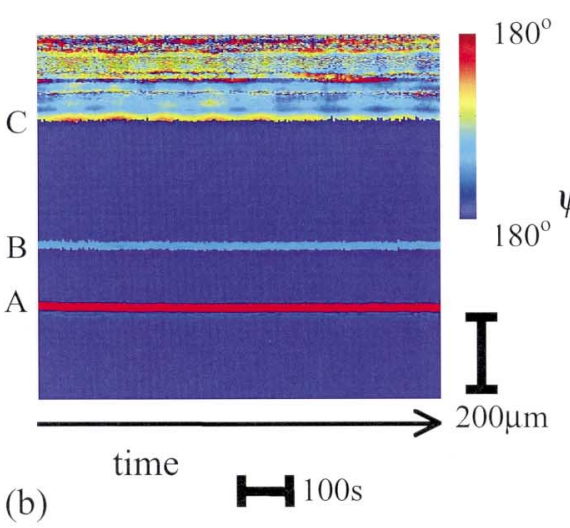

(b)

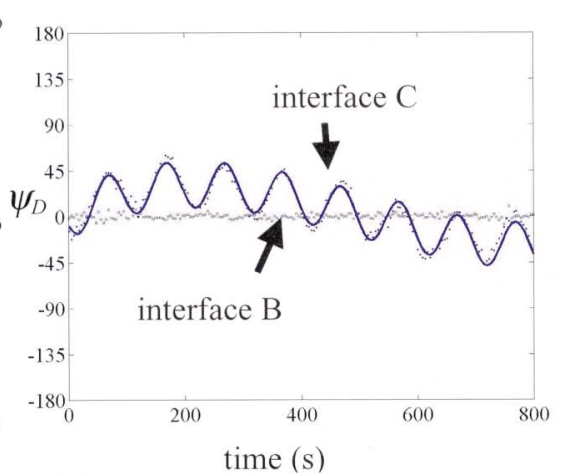

(c)

Fig. 2. PRI analysis of a slowly moving PZT: (a) geometrical arrangement, (b) depth-resolved PRI image of $\Psi_{D}(t)$, (c) trace of $\Psi_{D}(t)$ at the PZT surface and the second coverslip interface. The dashed curves in (a) indicate the beam profile. 


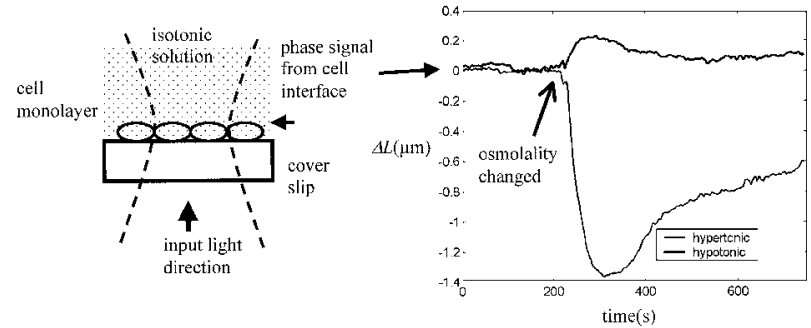

Fig. 3. PRI analysis of a live HT29 cell monolayer grown on a coverslip. The graph shows changes in cell thickness when the osmolality is changed from its normal value to $85 \%$ (hypotonic) and $115 \%$ (hypertonic) at $t=230 \mathrm{~s}$. The dashed curves indicate the beam profile.

sinusoidally with a period of $100 \mathrm{~s}$ by an applied voltage of amplitude of $2.25 \mathrm{~V}$. Figure 2(b) shows the time-dependent depth profile of $\psi_{D}(t)$ obtained from the system over $850 \mathrm{~s}$. Note the sinusoidal variations in phase at both the PZT surface (interface C) and beneath it, demonstrating the capability of depth resolution. Figure 2(c) is a plot of the time trace of $\psi_{D}(t)$ associated with interface C. For comparison, the time trace of $\psi_{D}(t)$ associated with the back surface of the coverslip (interface B) is also plotted. Analysis of $\psi_{D}(t)$ for the PZT surface reveals a period of $100.1 \pm 2.3 \mathrm{~s}$ and a peak-to-peak variation of $45.2 \pm 7.7^{\circ}$ (or equivalently a $36.6 \pm 6.2 \mathrm{~nm}$ displacement of the actuator). This experiment establishes that the system's motional sensitivity is $\sim 1 \mathrm{~nm} / \mathrm{s}$, the nominal speed of this motion. The slow drift in the PZT phase can be attributed to slow variations in the PZT-to-coverslip separation. This attribution of the drift to mechanical drifts in the sample as opposed to an error in the system is validated by the fact that $\psi_{D}(t)$ for the back surface of the coverslip is stationary, with an uncertainty of $\pm 4.5^{\circ}$ (or $\pm 3.6-\mathrm{nm}$ variation) over the entire scan.

We next apply the method to study the reaction of cell volume to hypotonic and hypertonic environmental changes. It was previously established by use of flow cytometry ${ }^{8}$ that cells respond to hypertonicity (hypotoncity) with an initial shrinking (swelling), followed by a compensatory, slower reversal of the initial responses. We perform a similar experiment with HT29 intestinal epithelial cell monolayers grown to confluence on coverslips. The cell monolayers are immersed in isotonic buffer (Hank's balanced salt solution). The index mismatch between the cells and the medium causes the top membrane surface to reflect light. ${ }^{9}$ We focus the low-coherence beam directly on the cell culture. The focal spot is $17 \mu \mathrm{m}$ wide, and the average cell diameter is $10 \mu \mathrm{m}$, indicating that only a few cells are sampled during the experiment. When the buffer's osmolality is abruptly changed to $85 \%$ (115\%) of its normal value, the phase shift $\psi_{D}$ associated with the cell membrane surface changes rapidly and then slowly recovers. In the hypotonic (hypertonic) case, the maximum change in cell thickness is $\sim 0.2 \mu \mathrm{m}$ $(-1.3 \mu \mathrm{m})$ (the refractive index of the cells is assumed to be 1.36), and the duration of the entire process is several hundred seconds [Fig. 3(b)]. The difference in response to the osmolality changes can be attributed to the different transport proteins involved in the recovery processes. To our knowledge, this is the first time that cell volume changes of such small magnitudes have been observed optically and continuously traced in situ for a few cells.

We have demonstrated that PRI is capable of measuring small and slow changes and that, in a biological context, it can be employed to study subcellular dynamics of a few cells or even parts of a single cell. The latter can be achieved simply by use of a tighter focal spot obtained with a higher-power microscope objective. As with all phase-based techniques, PRI has to contend with the problem of phase wrapping. This phase ambiguity sets a limit on the fastest detectable length change. At the present frame-refresh rate of $4.25 \mathrm{~s}$ and our low-coherence wavelength of $1550 \mathrm{~nm}$, this upper limit is $\sim 100 \mathrm{~nm} / \mathrm{s}$. However, there is no theoretical lower limit to the dynamics that can be measured with this technique. This consideration makes PRI a good complement to Doppler OCT, in that together they can potentially be used to measure motions over a wide range of magnitudes.

We acknowledge valuable discussions with $\mathrm{P}$. Knauf of the University of Rochester and C. Boone and I. Georgakoudi of the Massachusetts Institute of Technology (MIT) Laser Biomedical Research Center. This work was carried out at the MIT Laser Biomedical Research Center and supported by grants from the National Institutes of Health (P41-RR02594) and Hamamatsu Corporation. A. Wax is supported by a National Research Service Award from the National Institutes of Health. C. Yang's e-mail address is chyang@mit.edu.

*Present address, Department of Pathology, Children's Hospital, Boston, Massachusetts 02115.

\section{References}

1. S. L. Rogers and V. I. Gelfand, Curr. Opin. Cell Biol. 12, 57 (2000).

2. D. S. Coffey, J. P. Karr, R. G. Smith, and D. J. Tindall, Molecular and Cellular Biology of Prostate Cancer (Plenum, New York, 1991).

3. K. Strange, Cellular and Molecular Physiology of Cell Volume Regulation (CRC Press, Boca Raton, Fla., 1993).

4. A. W. Partin, J. S. Shoeniger, J. L. Mohler, and D. S. Coffey, Proc. Natl. Acad. Sci. USA 86, 1254 (1989).

5. Y. Zhao, Z. Chen, C. Saxer, S. Xiang, J. F. de Boer, and J. S. Nelson, Opt. Lett. 25, 114 (2000).

6. S. Yazdanfar, A. M. Rollins, and J. A. Izatt, Opt. Lett. 25, 1448 (2000).

7. C. Yang, A. Wax, R. R. Dasari, and M. S. Feld, Opt. Lett. (2001).

8. K. R. Hallows, C. H. Packman, and P. A. Knauf, Am. J. Physiol. 261, C1154 (1991).

9. The interference signal from this interface cannot be resolved from the signal arising from the boundary between the coverslip and the cells, as the optical path difference is smaller than the coherence length. Extraction of amplitude-based information is not possible; however, the phase shift associated with either interface can be found by measurement at sufficient displacements from the peak interference signal. 